\title{
BINOCULAR DEPTH PERCEPTION IN SMALL-ANGLE STRABISMUS*
}

\author{
BY \\ E. J. NAYLOR AND A. STANWORTH \\ Department of Ophthalmology, University of Manchester
}

MEASUREMENTS of the binocular depth perception in a series of patients who, after treatment for strabismus, had fusion and stereopsis have shown that the depth perception is often surprisingly poor (Naylor, Shannon, and Stanworth, 1956). This was the case even in patients who had a good functional result on cover test. The modified major amblyoscope (Stanworth, 1958) provides a more accurate assessment of small-angle strabismus than has hitherto been available, and the present investigation was therefore designed to determine whether or not these findings on the instrument explained the relatively poor depth perception.

\section{Material}

Binocular depth perception was measured in 66 of the eighty patients examined on the modified major amblyoscope by Stanworth and da Cunha (1959a, b), the cases omitted being mainly at the beginning and end of the series, when the apparatus for depth perception testing was not available. The ages of the patients ranged from 5 to 10 years.

The measurements with the modified major amblyoscope included an assessment of the deviation and prism ductions both with the full binocular prism stimulus and with the central ten degrees eliminated by a green circle. The angle of deviation recorded was the average of six readings.

\section{Measurement of Depth Perception}

The estimation of binocular depth perception in the absence of uniocular clues presents a problem which, in the case of young children, can be solved only by compromise. A method which has found favour with many workers dealing with older subjects is the presentation of two vertical rods against an illuminated background, the subject being required to judge when the rods appear to be equidistant from him. It is usual to have one fixed and one movable rod. The subject may adjust the position of the movable rod under continuous illumination by means of strings and pulleys until the rods appear to be at the same distance, or he may be required to specify the position of the movable rod when the field is exposed for a brief interval of time. The

* Received for publication November 26, 1958. 
former method is unsatisfactory, especially for children, in that it calls for a certain amount of manual dexterity in addition to visual judgment. With very few exceptions we have found that children of even 5 or 6 years of age have no hesitation in saying "Back" or "Front" to indicate the position of one rod with respect to the other and we have therefore adopted the latter method.

If the percentage of "Back" responses are plotted for different separations of the two rods, an S-shaped curve is obtained (Fig. 1). A mirror image of

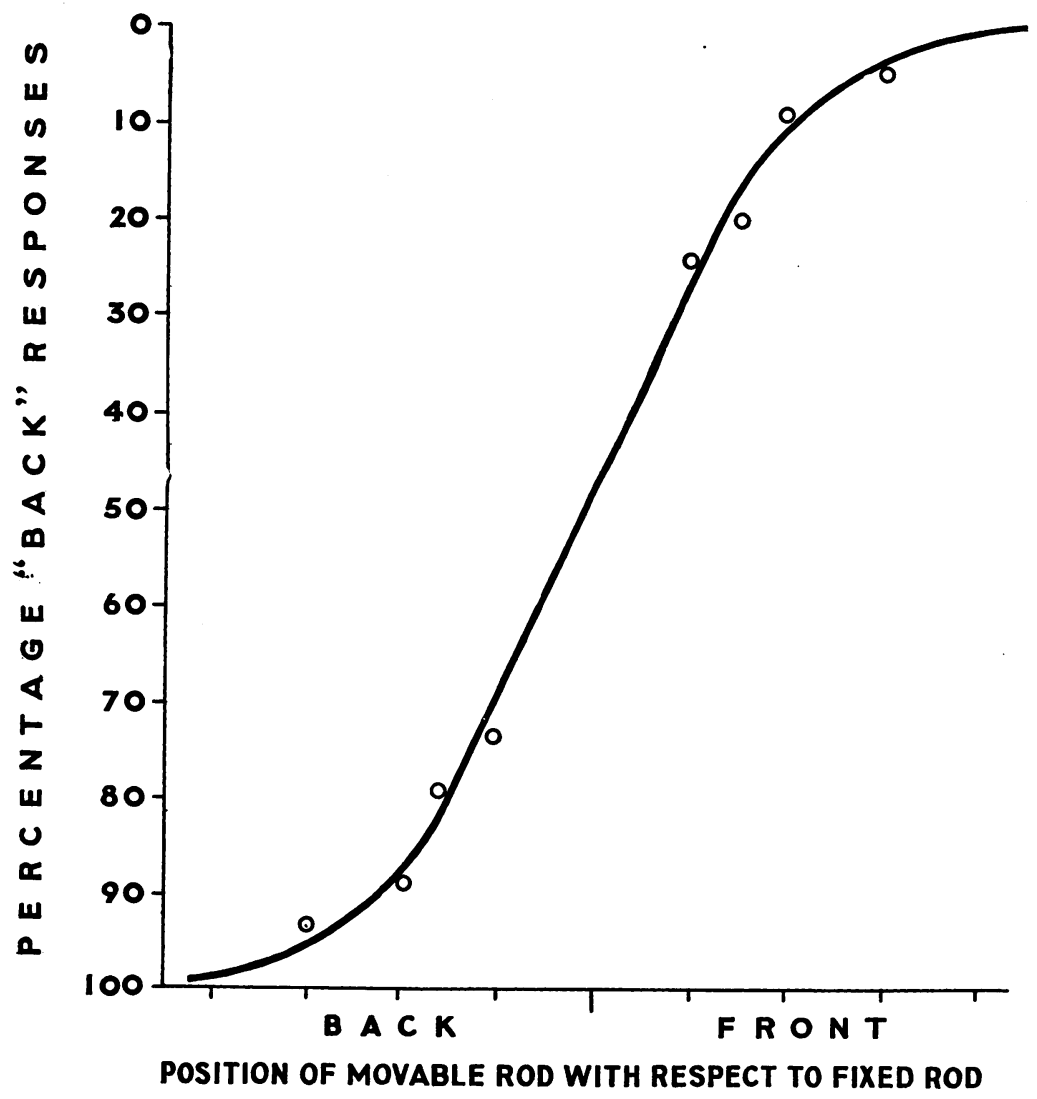

FIG. 1.-Relationship between responses and positions of rods.

this curve would be obtained if we had chosen to plot the percentage of "Front", instead of "Back" responses. In either case it is more convenient to change the ordinate scale so that the relationship between separation distance and responses is converted to a linear relationship. This may be accomplished by the probit method (see, for instance, Finney, 1952), which is theoretically desirable but is extremely time-consuming, and for our purpose 
has been found unnecessary except in a few cases. An alternative is to plot the responses on arithmetical probability paper as in Fig. 2, in which the position of the movable rod corresponding to the 50 per cent. level of "Back" responses gives the point at which this rod appears level with the fixed rod. In practice, this point will rarely coincide with the point at which the rods are physically equidistant from the patient. The acuity of depth perception is determined by the slope of the line; this is best specified by the distance between the points on the abscissa which correspond to the 84 per cent. and 16 per cent. levels on the ordinate. Half this distance is equivalent to the standard deviation of the distribution, and when expressed as the corresponding angle of binocular parallax, it gives a measure of the stereoscopic acuity.

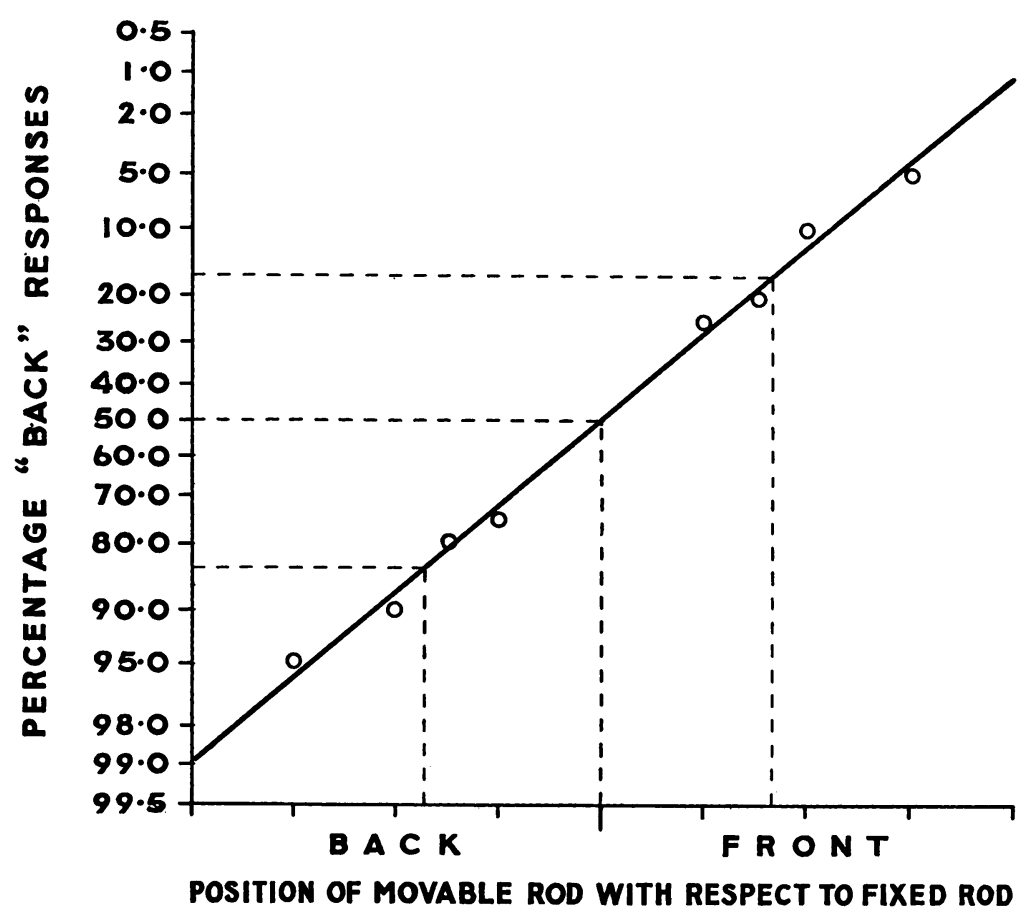

Fig. 2.-Same relationship as Fig. 1, plotted on probability paper.

\section{Testing Procedure}

The subject was comfortably seated in a darkened room, with his head supported by a chin and forehead rest, 3 metres from a rectangular aperture, $15 \times 6 \mathrm{~cm}$., in the front of a light-tight black box. The box contained a fixed, black, vertical rod, $3 \mathrm{~mm}$. in diameter, situated $15 \mathrm{~cm}$. from the aperture. A second similar rod could be moved to and fro along a scaled track situated either to left or right of the fixed rod at a distance of $6 \mathrm{~cm}$. from it. The rods 
were illuminated by a 150 -watt opal light bulb centrally situated behind a translucent plastic screen $30 \mathrm{~cm}$. from the front aperture. The light was controlled by an automatic time switch operating for a period of two seconds, so that when the light was switched on the subject saw only a black screen with an illuminated aperture revealing the silhouettes of the central portions of the rods, the extremities of which were not visible. During the time of illumination, the subject was required to estimate the relative positions of the rods and to indicate the position of the left hand rod with respect to the right hand one by simply saying "Back" or "Front". He was given three or four unrecorded trials to ensure that he understood what was required of him and to give the observer an approximate idea of the threshold distance.

The rods were then presented to him ten times each, front and back of the fixed rod, according to a previously determined random order, at a distance corresponding to the estimated threshold. As explained above, the threshold corresponds to about 84 per cent. of correct responses either front or back; if the subject obtained more than this number correct within the first series of twenty trials, the rods were then presented a further twenty times in similar fashion but with the movable rod positioned at a distance corresponding to a lower threshold (nearer to the fixed rod). Conversely, if the correct responses were much below 84 per cent. correct, the movable rod was positioned further away from the fixed rod before commencing the second series of twenty presentations. A third series of twenty presentations was made in the light of the responses obtained on the first two series, in order to get as many responses as possible near to the threshold value. A fourth series of twenty presentations was made if this seemed to be particularly indicated, but this was not a routine procedure since prolonging the testing tended to make the child less co-operative.

The results were tabulated, converted into percentages of "Back" responses, and plotted on probability paper, as previously described. The best fitting line was drawn independently by two observers and the threshold value taken as the mean of the values found by each. In the few cases where the individual findings were discrepant by more than about 10 seconds, an accurate threshold value was calculated by the probit method.

\section{Results}

The 66 patients included four in whom it was doubtful if a squint had ever been present, two with intermittent divergent strabismus, and seven with amblyopia resulting in a difference in visual acuity between the two eyes of more than two Snellen lines. The latter were classified separately since such amblyopia might be expected to influence the depth perception independently of the angle of deviation.

The remaining 53 patients were classified according to the findings on the modified major amblyoscope into four groups (Stanworth and da Cunha, 1959a, b). 
(A) 28 patients with no deviation when measured with the whole target:

(1) Eighteen with normal steady readings with whole target and green circle, indicating bifoveal fixation.

(2) Eight with a tendency to break down to manifest squint or to fixation disparity. Three of these had abnormally large readings with the green circle before the breakdown occurred.

(3) Two with abnormally large but reasonably steady readings with the green circle.

(B) 20 patients with fixation disparity:

(1) Fourteen with reasonably steady readings.

(2) Two with a tendency to break down to manifest squint with suppression.

(3) Four with marked suppression.

(C) Two patients with abnormal retinal correspondence.

(D) Three patients with manifest squint with suppression.

The depth perceptions of the patients in these various groups are shown in Fig. 3 (opposite), in steps of 25 seconds.

All the four patients in whom it was doubtful whether a squint had ever been present and the two patients with intermittent divergent strabismus gave normal readings on the modified major amblyoscope, indicating bifoveal fixation; five had depth perception of 25 seconds or better; the sixth had depth perception of 26 seconds.

Amblyopia had an obvious effect on depth perception, only one patient having depth perception better than 125 seconds; this patient (Stanworth and da Cunha, 1959a, Case 6) showed no deviation with the whole target, but broke down to manifest squint with suppression with the green circle; the visual acuity was $6 / 6$ in the right eye, and $6 / 18$ in the left, and the depth perception was about 50 seconds.

In assessing the depth perception in the remaining patients, it is obvious that a tendency to break down to manifest squint with suppression might in itself account for poor depth perception, since such a breakdown might occur at the beginning or during the carrying out of the test. The most valuable comparison is therefore between those patients with steady bifoveal fixation (Group A1) and those with steady fixation disparity (Group B1). In the former group, sixteen of the eighteen patients had depth perception better than 125 seconds, compared with only three of the fourteen patients in the latter group. The one patient with steady fixation disparity who had depth perception better than 75 seconds began with depth perception of about 35 seconds but then broke down to much worse than this. The average age at testing in both groups was between $7 \frac{1}{2}$ and 8 years, and the contrast between the groups still remains even if all children under 7 years are excluded. 


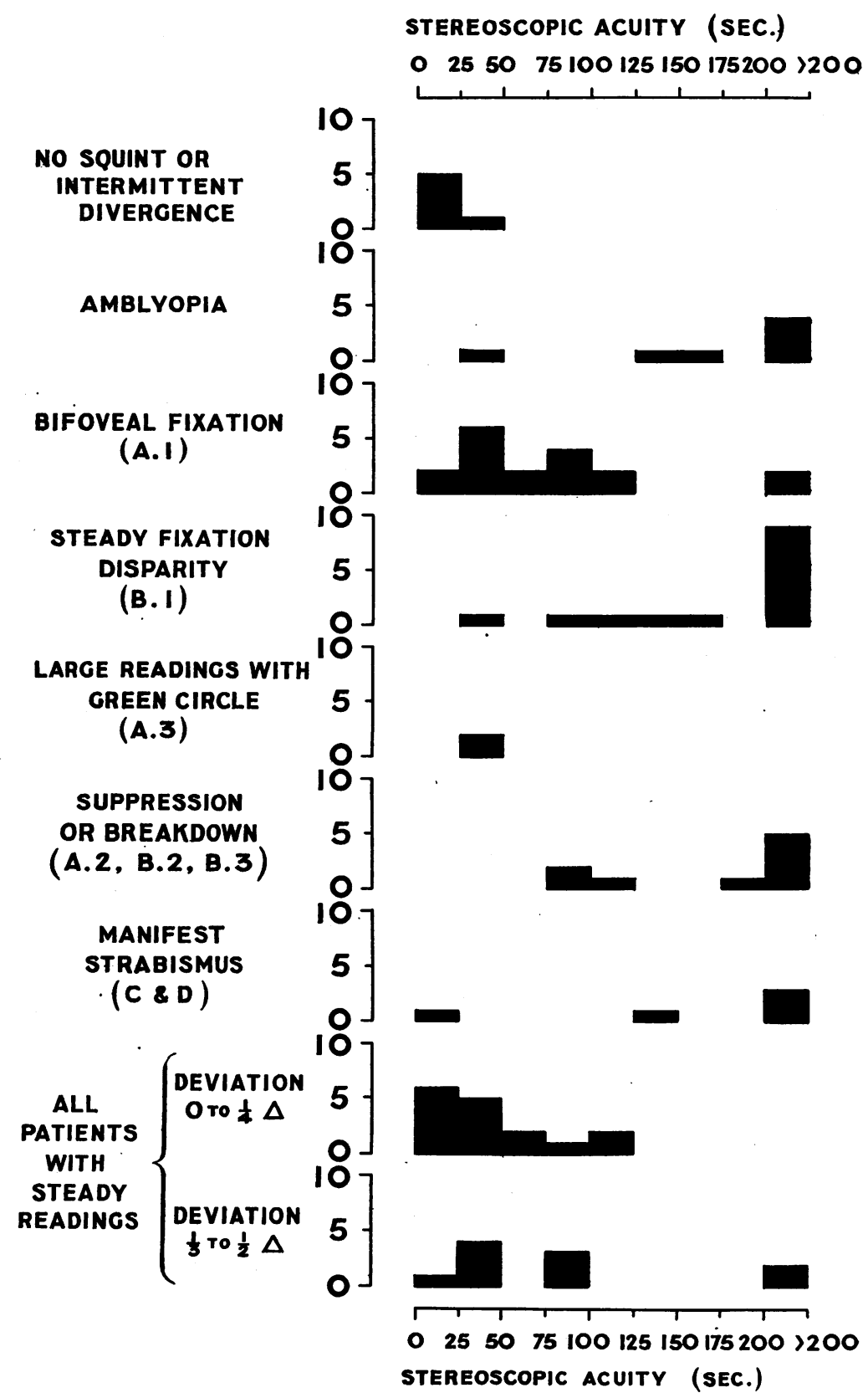

Fig. 3.-Depth perception in various types of strabismus.

Abscissa: Depth perception, grouped every 25 seconds.

Ordinates: Number of patients in each depth perception group. 
The two patients with abnormally large but reasonably steady readings with the green circle (Group A3) both had good depth perception (30 and 32 seconds), though one showed a tendency to break down during the measurements.

The patients who tended to show suppression or to break down to manifest strabismus on the modified major amblyoscope (Groups A2, B2, and B3) showed relatively poor depth perception, especially those who at best showed fixation disparity (Groups B2 and B3).

The patients with manifest squint, with or without abnormal retinal correspondence, all had poor depth perception except one; this was the only patient in the series in whom the modified major amblyoscope undoubtedly gave misleading readings, causing a breakdown to manifest strabismus in a patient who normally showed only esophoria, the depth perception being 12 seconds.

Even in patients with steady readings in the range we have taken to be normal with the whole target (Groups A1 and A3, together with those who had probably never had a squint, and those with intermittent divergent strabismus), there is a tendency for the depth perception to be better if the measured deviation on the modified amblyoscope is particularly small. All fourteen patients with a deviation of 0.25 prism dioptre or less had depth perception better than 125 seconds; six (38 per cent.) had depth perception of 25 seconds or better, compared with only one in ten patients with deviations of 0.33 to 0.5 dioptre.

\section{Discussion}

Two factors have to be remembered in interpreting these results. First, the depth perception in children cannot be expected to attain the same degree of precision as that in adults, since any wandering of the attention will result in an increase in the errors made and hence in the measured threshold. It is not surprising, therefore, that not all children with perfect binocular vision achieve a threshold of about 10 seconds such as one might expect in adults (Ogle, 1950), and thresholds of 25 seconds or even worse may be considered satisfactory. Secondly, the stimulus to fusion provided by the apparatus used to measure the depth perception is not particularly strong, so that any tendency for binocular vision to break down in the absence of a strong fusion stimulus will result in a relatively poor degree of depth perception under these conditions; such patients may in everyday life obtain more value from their binocular depth perception than would be apparent from the present results.

Nevertheless, the results show clearly that the depth perception is related to the angle as measured on the modified major amblyoscope. This is not surprising, for the ability of patients with fixation disparity to achieve binocular vision in the presence of a slight deviation is due to the capacity to fuse slightly disparate points; such fusion is normally used by subjects with bifoveal fixation to give depth perception, and its use merely to enable fusion 
to take place in the presence of a slight deviation must obviously interfere with its use for depth perception.

The fact that patients who show fixation disparity only with the green circle seem to have good depth perception, together with such patients' relatively normal cover tests (Stanworth and da Cunha, 1959b), suggests that the readings with the whole target are a reliable indication of the position of the eyes, and are not due to the conditions of the test; the difference between the readings with and without the green circle are therefore likely to be true differences in the amount of disparity occurring under the two conditions.

The fact that almost all the patients with normal readings with the whole target had reasonably good depth perception, whereas those with abnormal retinal correspondence had poor depth perception, suggests that it is unlikely that abnormal correspondence is being overlooked in any substantial number of patients.

The finding that depth perception appears to be correlated to some extent with average deviations of less than 0.5 prism dioptre obviously requires further investigation. The measurements in this series were carried out on a prototype model of the modified major amblyoscope, and it is hoped that greater precision will be possible with the more accurate instrument now available.

\section{Summary}

66 patients were examined with the modified major amblyoscope and their binocular depth perception determined. It is shown that there was a reasonably close correspondence between the angle of deviation and the binocular depth perception, patients with fixation disparity having relatively poor depth perception.

We are indebted to Miss $\mathrm{D}$. da Cunha for carrying out the majority of the readings with the modified major amblyoscope.

\section{REFERENCES}

FinNex, D. J. (1952). " "Probit Analysis", 2nd ed. University Press, Cambridge.

Naylor, E. J., Shannon, T. E., and Stanworth, A. (1956). Brit. J. Ophthal., 40, 641.

OGLE, K. N. (1950). "Researches in Binocular Vision", p. 137. Saunders, Philadelphia.

StANWORTH, A. (1958). Brit. J. Ophthal., 42, 270. and DA CUNHA, D. (1959a). Ibid., 43, 648. - (1959b). Brit. orthopt. J., 16, 85. 